\title{
Rapid Analysis Procedures for Triglycerides and Fatty Acids as Pentyl and Phenethyl Esters for the Detection of Butter Adulteration Using Chromatographic Techniques
}

\author{
Daniele Naviglio, ${ }^{1}$ Marina Dellagreca, ${ }^{1}$ Francesco Ruffo, ${ }^{1}$ \\ Anna Andolfi, ${ }^{1}$ and Monica Gallo ${ }^{2}$ \\ ${ }^{1}$ Department of Chemical Sciences, University of Naples Federico II, Via Cintia 4, 80126 Naples, Italy \\ ${ }^{2}$ Department of Molecular Medicine and Medical Biotechnology, University of Naples Federico II, Via Pansini 5, \\ 80131 Naples, Italy \\ Correspondence should be addressed to Monica Gallo; mongallo@unina.it
}

Received 31 July 2016; Revised 16 October 2016; Accepted 8 November 2016; Published 6 April 2017

Academic Editor: Marina Carcea

Copyright (C) 2017 Daniele Naviglio et al. This is an open access article distributed under the Creative Commons Attribution License, which permits unrestricted use, distribution, and reproduction in any medium, provided the original work is properly cited.

\begin{abstract}
This paper presents the development of three methods for quality control, fraud detection, and authentication of butter fat and other oils/fats using chromatographic techniques, with one method for triglycerides and two methods for fatty acids (FAs). The procedure for the analysis of triglycerides requires only dissolution of the sample in $n$-hexane and gas chromatography (GC) analysis using a capillary column. The second method is based on the transesterification of triglycerides as pentyl esters in a singlestep reaction using sodium pentanoate in pentanol. The reaction proceeds at room temperature and is similar to the potassium hydroxide-catalysed transesterification of triglycerides with methanol and even more similar to the sodium methoxide method and sodium butanoate method. The advantage of using pentyl esters includes reducing the volatility of short-chain FAs, and substantial recoveries were obtained compared with methyl ester analysis. The third method involves the transesterification of triglycerides in fat through reaction with 2-phenylethanol in a single step; 2-phenylethanol possesses a chromophore, and the phenethyl esters formed are analysed by high-performance liquid chromatography (HPLC) with UV detection.
\end{abstract}

\section{Introduction}

In the human diet, the fatty acids (FAs) of vegetable and animal lipids (in addition to carbohydrates and proteins) play an important role, contributing to structural function and providing energy. Vegetable- and animal-based foods contain fats that naturally include various amounts of different FAs [1]. Currently, despite the regulation of every type of food, food fraud is very common, especially with respect to higher quality foods of greater value, such as milk and its derivatives. Therefore, methods to detect foreign fats in milk are needed to prevent the declaration of goods as pure fat milk products, when they may contain cheaper vegetable or animal fats. In the food industry, the FA composition of milk is used as a food fraud indicator because it can reveal the presence of foreign fats in mixtures made with cow's milkfat. Many works have addressed the identification and investigation of fatty substances because of the increased availability of survey methods that are sufficiently powerful and sensitive, such as general chromatographic techniques and specifically gas chromatography (GC). Therefore, GC analysis, first, and the Precht method, later, helped to identify the types of foreign animal fats added to the composition of authentic butter obtained from cow's milk through separation of the constituents of the lipid matrix, specifically triglycerides, providing a measure of the butter authenticity. The Precht method was the EU's official analysis method for the recognition of foreign fats and is based on the GC separation of triglycerides on a packed column, which does not separate individual triglyceride peaks but rather peaks 
corresponding to triglyceride families [2, 3]. This method was updated to utilize capillary columns as packed columns became outdated.

In recent years, several alternative methods to the official Precht method have been developed to utilize short capillary columns possessing apolar stationary phases, which provide the same results as the packed columns. Furthermore, the use of short, narrow-bore columns results in higher carriergas velocity and consequently optimal performance in terms of separation based on triglyceride families and very short elution programmes for triglycerides [4-7].

Reversed-phase high-performance liquid chromatography (RP-HPLC) with evaporative light scattering detection (ELSD), which facilitates the excellent separation of triacylglycerols, has been developed to analyse oils and fats [8]. In food research, triglyceride analysis has been used to study crystallization phenomena to detect the adulteration of fats and oils in cocoa butter [9], milkfat [10], and olive oil [11] to identify virgin olive oils from a single cultivar [12] or a protected designation of origin [13].

For triglyceride analysis, the sample of fat or oil is dissolved in an organic solvent, such as hexane, and then submitted for analysis. For FA analysis, a transesterification reaction, in which the FAs are converted into their more volatile methyl esters, is required [14].

Eras et al. [15] employed chlorotrimethylsilane (CTMS), a toxic halide based on silicon [15] that can be used as a reagent to transform triglycerides into volatile fatty esters. Other methods of triglyceride transesterification include the use of catalysts, such as boron trifluoride in methanol $[16,17]$.

Some reported analysis procedures convert triglycerides into picolinyl esters, $N$-acyl pyrrolidines, and DMOX derivatives (4,4-dimethyloxazoline) to enable detection by mass spectrometry. These derivatives are separated using a lowpolarity column because of its high thermal stability [18]. Gas chromatography-mass spectrometry (GC-MS) has been widely used for the structural analysis of FAs. However, the identification and localization of some structural features, such as hydroxyl groups, epoxy groups, branched chains, rings, and double bonds, are problematic. The derivatization of triglycerides as FA methyl esters could be used to identify certain types of branching but not additional methyl branches, double bonds, or other types of unsaturation because the structural information obtained from the mass spectra of functionalized unsaturated FA methyl esters is typically insufficient [19]. The generated spectra do not provide sufficient information about these structures [20, 21], primarily because of the ionization of the double bonds in unsaturated FAs that occurs during electron impact [22].

To overcome the migration problem, the carboxyl group can be reacted with a nitrogen compound to form an amide bond. The resulting derivatives have many sites with a positive charge (the electronic charge captured during ionization) that minimize the ionization of the double bond and migration. The first nitrogen derivative used is $\mathrm{N}$-acyl pyrrolidine [23].

However, most researchers currently prefer picolinyl esters or derivatives of DMOX [24-29]. A major challenge in the development of chromatographic methods for this separation has been the rational choice of optimal experimental conditions that provide a suitable solution in a reasonable amount of time. Computer simulation is a valuable tool for the systematic optimization of these separations and varying parameters such as the initial temperature and the temperature programme [30].

Given the importance of FAs in foods and their health implications, this work considered two widely consumed foodstuffs, milk and oil. These foods possess similar triglycerides (TAG), which are essential for supplying FAs in the human body. The goal of this work was to assess three analysis methods for fats (mainly triglycerides) that are rapid and inexpensive and constitute valid alternatives to more sophisticated systems, such as liquid chromatography/electrospray ionization-mass spectrometry (LC/ESIMS) [31], two-dimensional liquid chromatography/gas chromatography (LCxGC) [32], or silver reversed-phase and silver ion high-performance liquid chromatography-mass spectrometry (RF-HPLC-MS) [33]. As stated above, the screening methods that are currently used to study fats are based on analysing triglycerides in terms of the triglyceride distribution in fat and analysing FAs after esterification with glycerol and release via transesterification.

\section{Materials and Methods}

2.1. Samples, Reagents, and Standards. Samples of milk and butter were of the same brand and purchased from the same dealer, and their authenticity was verified. The solvents used were $n$-hexane, $n$-pentane, 1 -pentanol, methanol, and acetonitrile; HPLC grade was purchased from Merck (Darmstadt, Germany). Diethyl ether and dichloromethane were analytical grade and purchased from Carlo Erba (Milan, Italy). Ultrapure water was obtained from a Milli-Q system (Millipore, Billerica, MA, USA). All reagents (2-phenylethanol, sodium metal, potassium hydroxide, $1 \mathrm{~N}$ hydrochloric acid, and anhydrous sodium sulphate) were analytical grade and purchased from Fluka (Buchs, Switzerland). Hydrogen, air, and nitrogen cylinders were purchased from Sol (Milan, Italy).

2.2. Instrumentation. A GC (Perkin Elmer AutoSystem XL) was equipped with a programmable split-splitless (PSS) injector and flame ionization detector (FID) (Perkin Elmer, Norwalk, CT, USA). Data were acquired using Turbochrom software, version 4.1. Another GC (DANI GC-1000, DANI, Monza, Italy) was equipped with a programmable temperature vaporization (PTV) injector and an FID connected to an integrator (model $8890 \mathrm{HP}$, Hewlett-Packard, Palo Alto, CA, USA). An LC (Shimadzu SCL 6A) was connected to an SPD-6 UV-VIS detector with an electronic integrator (CR6A, Shimadzu, Tokyo, Japan). The centrifuge used was PK 131 (ALC International, Milan, Italy).

2.3. GC Analysis of Triglycerides: Sample Preparation. Approximately $50 \mathrm{mg}$ of anhydrous fat was dissolved in $1.0 \mathrm{~mL}$ of hexane, and $1 \mu \mathrm{L}$ of this mixture was injected directly into 
the GC. When the fat contained a certain percentage of water (butter), a drying procedure was used. First, $10 \mathrm{~g}$ of butter was melted at $60^{\circ} \mathrm{C}$ on medium-porosity filter paper with anhydrous sodium sulphate, and $50 \mathrm{mg}$ of the dried sample was dissolved in $1 \mathrm{~mL}$ of hexane; then, $1 \mu \mathrm{L}$ of this solution was injected into the GC.

Separation was achieved on an RTX 65-TG capillary column with a $65 \%$ phenyl methyl silicone HT (TG) stationary phase (Restek, Bellefonte, CA, USA) $(l=30$ m, i.d. $=0.25 \mathrm{~mm}$, and $\mathrm{ft}=0.25 \mu \mathrm{m}$ ). The injector temperature was programmed as follows: $60^{\circ} \mathrm{C}$ was maintained for $12 \mathrm{sec}$, followed by an increase at $999^{\circ} \mathrm{C} / \mathrm{min}$ to $370^{\circ} \mathrm{C}$ and a $5 \mathrm{~min}$ hold. The oven temperature was programmed as follows: $250^{\circ} \mathrm{C}$ was maintained for $2 \mathrm{~min}$, followed by an increase at $5^{\circ} \mathrm{C} / \mathrm{min}$ to $360^{\circ} \mathrm{C}$ and a $4 \mathrm{~min}$ hold. The detector temperature was $370^{\circ} \mathrm{C}$. Hydrogen was used as the carrier gas at a flow rate of $1.5 \mathrm{~mL} / \mathrm{min}$. The split ratio was $1: 80$.

2.4. GC Analysis of Pentyl Esters of FAs. Approximately $50 \mathrm{mg}$ of anhydrous fat or oil was weighed and added to $1 \mathrm{~mL}$ of $n$-pentane. The mixture was stirred to completely dissolve the fat, then, added to $200 \mu \mathrm{L}$ of $2 \mathrm{~N}$ sodium pentoxide in pentanol, and stirred for $2 \mathrm{~min}$. Then, $400 \mu \mathrm{L}$ of $1 \mathrm{~N} \mathrm{HCl}$ was added, and the mixture was shaken for $30 \mathrm{sec}$ and centrifuged at 448 RCF (relative centrifugal force) for $1 \mathrm{~min}$. Subsequently, $0.5 \mu \mathrm{L}$ of the organic phase (upper layer) was injected into the GC.

To prepare the sodium pentoxide solution, $2 \mathrm{~g}$ of metallic sodium was weighed under a hood and placed in anhydrous $n$-pentane to remove the surface residues of kerosene. The metallic sodium was transferred to a dark glass bottle containing $100 \mathrm{~mL}$ of pentanol. To completely react the sodium metal, the bottle was left open to evacuate the hydrogen produced.

GC analysis of the FA pentyl esters was performed on a column with a $90 \%$ bis-cyanopropyl phenyl silicone stationary phase (Restek, Bellefonte, CA, USA) $(l=50 \mathrm{~m}$, i.d. $=0.25 \mathrm{~mm}$, and $\mathrm{ft}=0.25 \mu \mathrm{m}$ ) to analyse the methyl esters of the FAs. The injector temperature was programmed as follows: $50^{\circ} \mathrm{C}$ was maintained for $15 \mathrm{sec}$, followed by an increase at $999^{\circ} \mathrm{C} / \mathrm{min}$ to $270^{\circ} \mathrm{C}$ and a $3 \mathrm{~min}$ hold. The column-temperature programming was as follows: $70^{\circ} \mathrm{C}$ was maintained for $2 \mathrm{~min}$, followed by an increase at $8^{\circ} \mathrm{C} / \mathrm{min}$ to $250^{\circ} \mathrm{C}$ and a $3 \mathrm{~min}$ hold. The detector temperature was $270^{\circ} \mathrm{C}$. Hydrogen was used as the carrier gas at a flow rate of $2 \mathrm{~mL} / \mathrm{min}$. The split ratio was $1: 80$.

2.5. Transesterification of Triglycerides as Phenethyl Esters Using HPLC. Approximately $50 \mathrm{mg}$ of anhydrous fat or oil was weighed, $1 \mathrm{~mL}$ of anhydrous $n$-pentane was added, and the mixture was shaken to completely dissolve the fat. Approximately $200 \mu \mathrm{L}$ of the transesterification reagent, phenethyl ethanoate in phenyl ethanol, was added, and the mixture was stirred for $2 \mathrm{~min}$.

Approximately $400 \mu \mathrm{L}$ of $1 \mathrm{~N}$ hydrochloric acid was added, and the mixture was stirred for $1 \mathrm{~min}$ and then centrifuged at 448 RCF for $2 \mathrm{~min}$. The upper phase was transferred into a vial and $n$-pentane was evaporated under

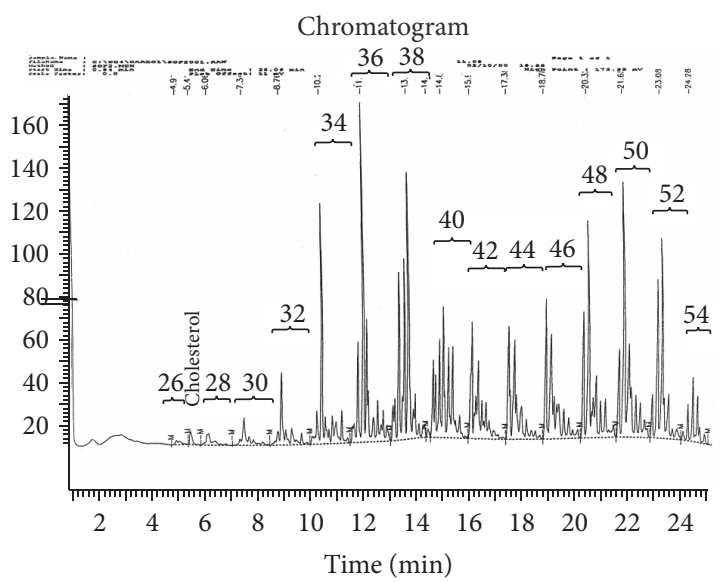

FIGURE 1: Gas chromatogram showing a typical integration for 15 families of triglycerides from 26 to 54 carbon atoms, excluding the contribution of the glycerol moiety. The cholesterol peak is highlighted.

a gentle stream of nitrogen. Then, $2 \mathrm{~mL}$ of acetonitrile was added, and the mixture was stirred to completely solubilize the phenethyl esters. Then, $20 \mu \mathrm{L}$ of this solution was injected into the HPLC.

To prepare the sodium phenethyl oxide solution, $2 \mathrm{~g}$ of metallic sodium was weighed and placed in anhydrous $n$ pentane to remove the surface residues of kerosene. Then, the metallic sodium was transferred to a dark glass bottle to which $100 \mathrm{~mL}$ of phenethyl ethanol had been previously added. To completely react with the metallic sodium, the bottle was left open to evacuate the hydrogen produced. The use of metallic sodium required all proper precautions and operation under the hood.

Separation was achieved on a Luna reversed-phase column with a phenyl hexyl silicone stationary phase (Phenomenex, Torrance, CA, USA $(l=250 \mathrm{~mm}$, i.d. $=4.6 \mathrm{~mm}$, and porosity $=5 \mu \mathrm{m}$ ). The elution programme (linear gradient) was as follows: phase A, $65 \%-35 \%$ acetonitrile in water; phase B, $90 \%-10 \%$ acetonitrile in dichloromethane; initial time ( $t=0 \mathrm{~min}), B=20 \%$; and final time $(t=20 \mathrm{~min}), B=$ $80 \%$. Spectrophotometric detection occurred at a wavelength of $258 \mathrm{~nm}$. The mobile phase flow rate was $1 \mathrm{~mL} / \mathrm{min}$.

\section{Results and Discussion}

3.1. Triglyceride Analysis of Butter Using a Capillary Column. The GC conditions were determined from analysis of anhydrous butter with a broad distribution of triglycerides, and the conditions obtained were then used for all other fats and oils.

In a typical gas chromatogram of the triglycerides in butter (chromatogram not shown), the integration function of the software yielded a quantitative estimate of the triglyceride components that satisfied two different criteria. The first criterion was the integration of triglycerides with the same number of carbons (TCN: theoretical carbon number) and divided families. Figure 1 shows a typical integration 


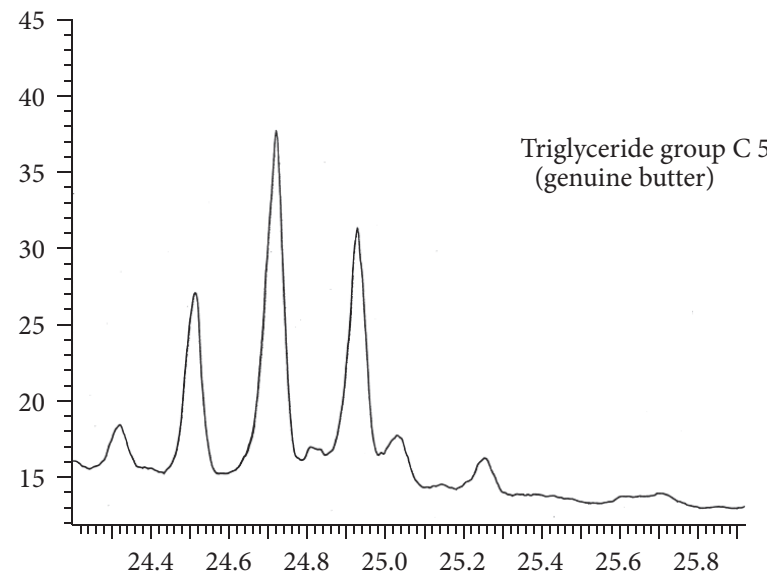

(a)

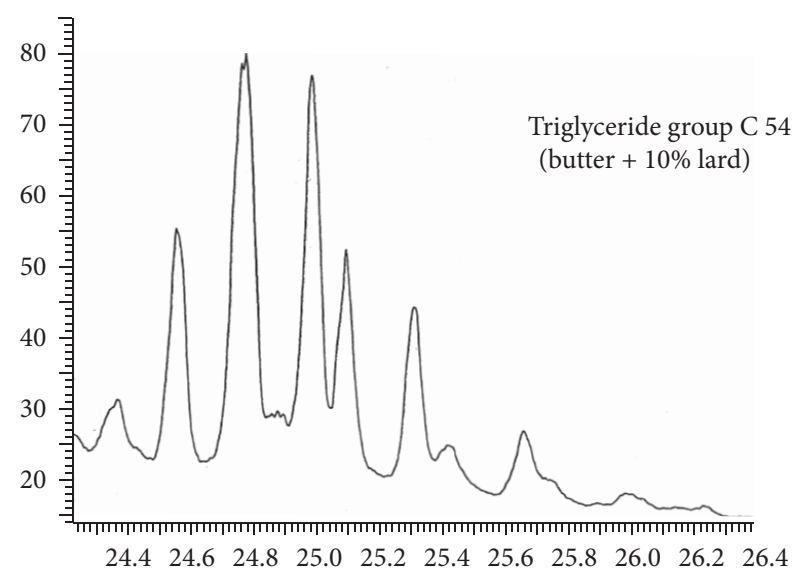

(b)

FIGURE 2: Gas chromatograms of the C54 family in authentic butter (a) and of the same family after the addition of 10\% (w/w) lard (b).

for individual groups or families of triglycerides, and 15 families were distinguished, ranging from a minimum of 26 to a maximum of 54 carbon atoms without the contribution of the carbon in glycerol. The cholesterol peak was also well separated and quantified in butter. Cholesterol is a sterol with especially important role in the physiology of animals and therefore humans, so obtaining a chromatogram in which cholesterol is identified as a separate quantifiable peak from the triglyceride peaks is useful. In the separation of triglycerides, we were able to separate the cholesterol peak in the same gas chromatogram, which is useful for quantification in butter oil and in lard. The integration method produced results comparable to those reported by Precht [2]. The second criterion was the integration of each peak representing a single triglyceride, even if the peak was only partially separated, to take advantage of the vertical integration (line drop) from a minimum number of points and by projecting the signal on the baseline. In summary, the first calculation criterion allowed triglycerides with the same number of carbons (TCN) to be divided into families using the integration for families, and the second criterion involved the integration of single triglyceride peaks.

Triglyceride families were identified for comparison with the data reported in the literature. However, such investigations typically include the identification of each individual peak using more sophisticated techniques, such as GC-MS. Finally, it was possible to separate triglycerides with the same number of carbon atoms, but this separation does not establish the stereochemistry of naturally occurring triglycerides.

\subsection{Analysis of the Integration Results via Two Methods.} Integration of the triglycerides in each family was used to detect the adulteration of butter using the Precht method [2]. The use of a polar column has two main advantages: (1) simple operation because of the milder GC conditions for immediate thermal equilibrium of the column and (2) potential increase of triglyceride compositional information obtained from the analysed sample. This separation of triglycerides added new information and provided an opportunity to determine whether each component had undergone changes because of the addition of other fats, as well as increasing the speed of the analysis. Figure 2 shows the chromatographic profile of the family of triglycerides with 54 carbon atoms in authentic butter (Figure 2(a)) and the same family after addition of $10 \%$ of lard (Figure 2(b)). Figure 2(b) is slightly shifted with respect to Figure 2(a), but comparison of the known peaks is possible, although some peaks are unidentified.

Figure 3 shows the gas chromatogram (a) of the C54 family in authentic butter, and the gas chromatograms in (b), (c), and (d) show the same family after the addition of small quantities of olive oil (between 1 and 3\% (w/w)). The threedimensional image shows that the ratio between peaks 3 and 4 was greater than the ratio with peak 1 in the butter; therefore, the addition of olive oil at $2 \%$ is optimal for the detection of adulteration.

3.3. Comparative GC Methods: Graphical Method. Analysis of the GC profiles and the overlaps obtained using the acquisition software revealed that, in all the cases analysed, the distribution was bimodal (double inverted bell) and the peak maxima of the two bells appeared in the C36 and C50 triglyceride families, respectively. The highest peak of C34 was always lower than that of C38; the highest peak of C48 was always higher than that of C52; and the separation between the two distributions always started at $\mathrm{C} 44$, where a minimum in the ideal curve that linked the two distributions through the maxima occurred (Figure 1). This distribution is relative to authentic butter and allows evaluation of the absence of adulteration (maximal).

To obtain a rapid analysis and preliminary assessment of the authenticity of the butter, a simple graphical overlay was constructed. The graphical comparison between the butter sample and the reference sample helped to exclude the possibility of an adulteration value of approximately $5 \%$, which was the minimum threshold for any type of added fat. As shown in Figure 4, the adulteration of butter with $10 \%$ lard resulted in a significant change in the GC profile of pure butter (chromatogram not shown). This adulteration 


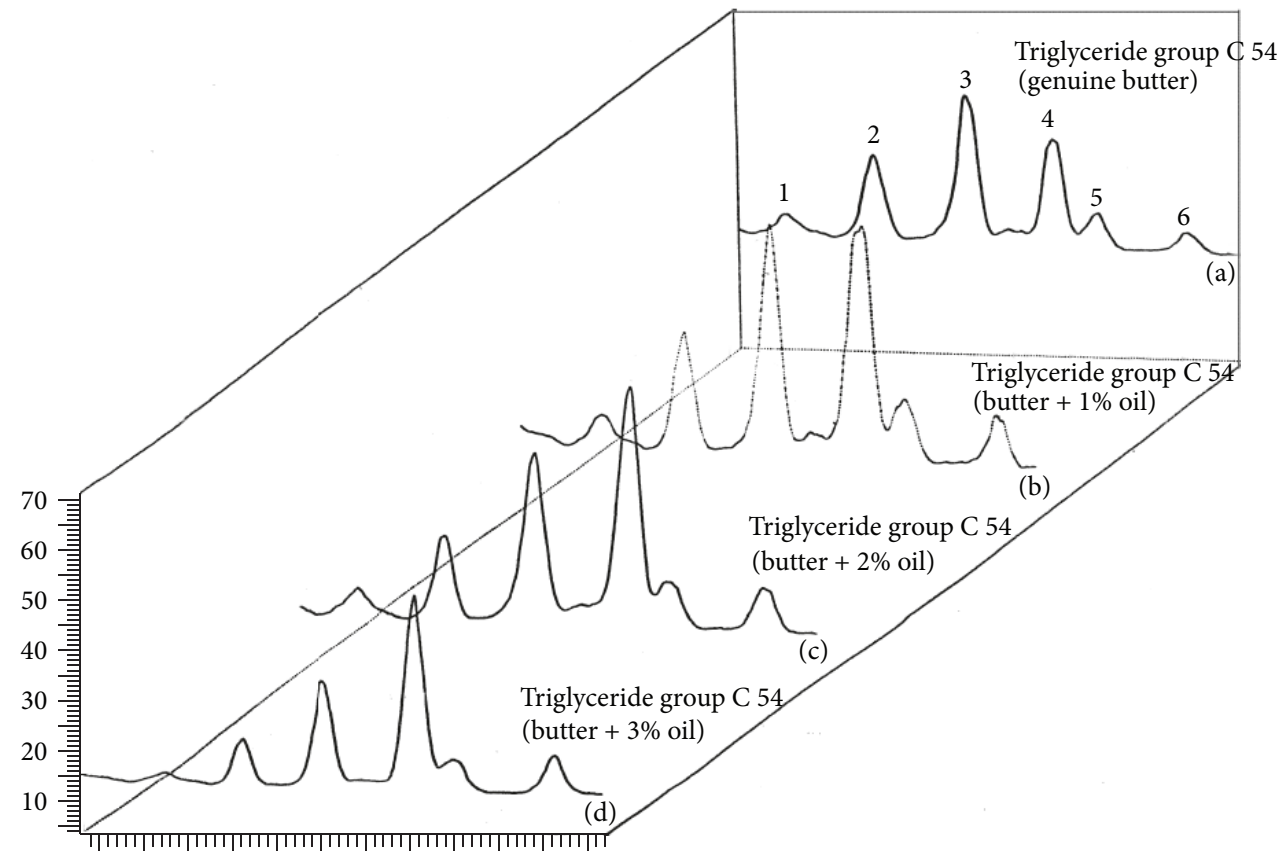

Figure 3: Gas chromatogram of the C54 family in authentic butter (a) and butter containing 1.02\% (b), 2.24\% (c), and 3.29\% (d) (w/w) of added olive oil.

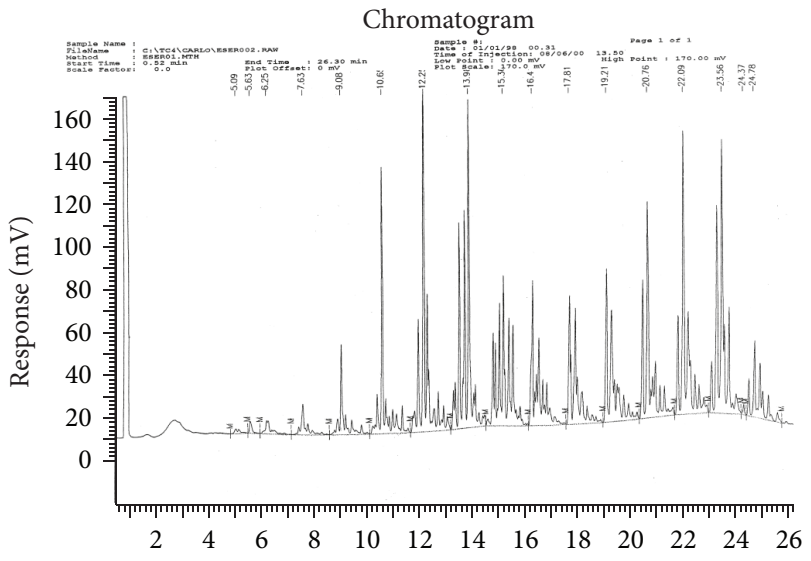

Figure 4: GC profile of authentic butter supplemented with $10 \%$ $(\mathrm{w} / \mathrm{w})$ lard.

was particularly evident for triglyceride families with high molecular weights.

Figure 2 compares the C54 family in authentic butter (Figure 2(a)) and adulterated butter (Figure 2(b)) containing approximately $10 \%$ lard. The simple overlap of the two profiles readily revealed the adulteration in the lower profile, in which the presence of three typical lard peaks at retention times of 25.1, 25.3, and $25.7 \mathrm{~min}$ is evident. With 5\%-3\% adulteration, the comparison did not provide an accurate or reliable response. Thus, depending on the nature of the fat added, the graphical method exhibited a different degree of sensitivity. For example, if a small amount of vegetable oil was added, a change in the macroscopic distribution of triglycerides was observed relative to C54 (as shown in Figure 2, which is a magnification of Figure 4).

Figure 3 shows the C54 family in authentic butter (curve (a)) in which we observed the characteristic profile of peaks 1 , $2,3,4,5$, and 6 . The addition of olive oil $(1.02 \%(\mathrm{w} / \mathrm{w}))$ resulted in an appreciable variation of this profile (curve (b)). Curves (c) and (d) show GC profiles of butter containing $2.24 \%$ and $3.29 \%$ added oil, respectively.

Increasing the percentage of vegetable oil in the butter gradually increased the difference between the relative areas of the peaks and specifically decreased the ratio of peak 3 (corresponding to tripalmitin) and peak 4 (corresponding to triolein), depending on the degree of adulteration. Thus, the relationship between the peaks and the addition of added fats in the butter could be determined. In all analysed cases, this ratio exceeded 1 for pure butter and decreased with the addition of other fats, such as $2 \%$ and $3 \%(\mathrm{w} / \mathrm{w})$ olive oil. This ratio exhibited a linear trend, and interpolations were possible (data not shown). Regarding the fraudulent addition of other fats to butter, such as 5\% or less lard, we observed no appreciable change in the macroscopic triglyceride profile. These data are not shown, but we detected adulteration from $2 \%$ lard in previous experiments of adding butter oils, similar to the adulteration of butter oil with vegetable oils at a concentration of $2 \%$.

A further example of the application of the graphical method includes comparing butter types from different backgrounds with the standard butter EEC BCR (Community Bureau of Reference) 164. As observed in Figures 5(a), 5(b), 5(c), and 5(d), graphical comparison of the 4 gas chromatograms via overlapping revealed that the compositions of the different butter types were the same. 


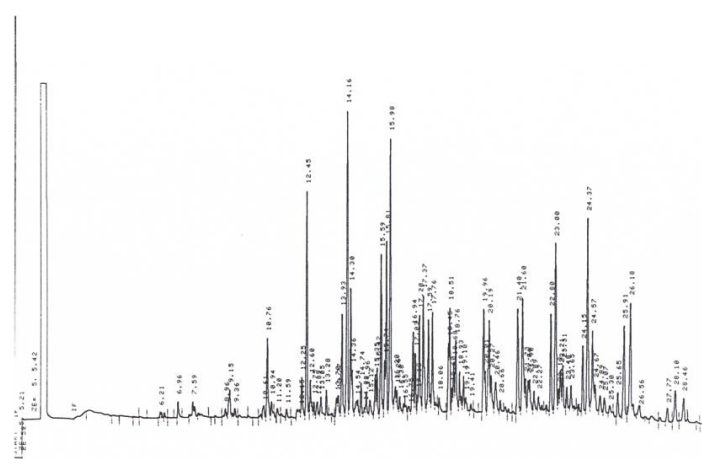

(a)

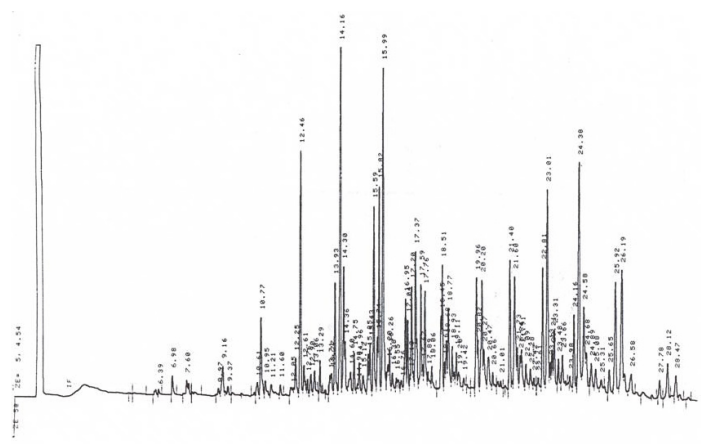

(c)

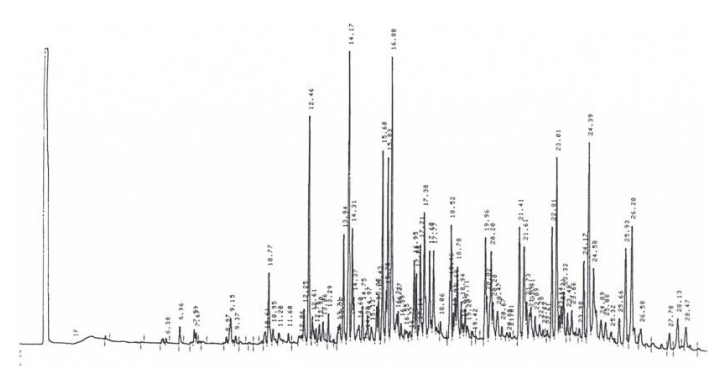

(b)

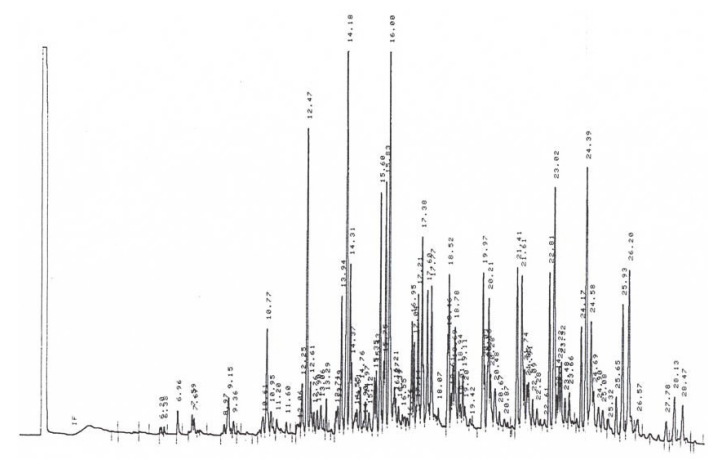

(d)

FIGURE 5: (a) Triglyceride profile of standard butter EEC (BCR 164); (b) triglyceride profile of Italian butter; (c) triglyceride profile of French butter; and (d) triglyceride profile of Dutch butter.

3.4. Mathematical Method. A method for further evaluation of the authenticity of butter involves applying mathematical methods to derive quantitative results for the triglyceride composition. The data can be analysed in two different ways: (1) the Precht method or (2) the individual evaluation of each integrated peak. Using the latter method, the individual triglycerides in fats can be compared.

3.5. Triglyceride Analysis to Determine the Authenticity of Butter (Precht Method). The Precht method employs a packed column and is based on the chromatographic separation of triglycerides into groups or families of compounds with the same total number of carbon atoms (TNC). This method requires the use of packed columns, which are obsolete and have been replaced by capillary columns. The Precht method has been thoroughly demonstrated in the literature and is essentially based on the comparison of a butter sample with a reference sample [2]. Using the mathematical Precht method, the authenticity of the butter can be assessed based on the separation of triglycerides on a capillary column with a $65 \%$ phenyl methyl silicone stationary phase, followed by integration of the individual triglyceride families.

To calculate the correct triglyceride concentration in the samples, the percentage value (area of the triglyceride family with respect to the total area, multiplied by 100) must be multiplied by the corresponding response coefficient (ratio between the average value obtained from the analysis of three replicates of a standard sample with a known triglyceride composition and the provided composition). The present study has summarized the results of previous studies that analysed 400 butter samples. The results obtained for the individual triglycerides were inserted into the appropriate formulas (identified by Precht) used for the detection of foreign vegetable and animal fats to obtain the index " $R$." The Precht method is essentially based on a comparison of the butter sample in the analysis with the reference butter, applying the criterion of integration for the families of triglycerides. By applying the formulas given by Precht, laborious calculations yield an index " $R$ " that is subsequently transformed by increasing the percentage of adulteration. Moreover, comparison of the integration of single triglyceride peaks is proposed to detect adulteration in butter oil. The results of the analysis of 400 authentic butters revealed that the values of " $R$ " obtained are within the range when using the Precht method [2] and taking standard butter as a reference (data not shown). This method utilizes the average weights of each triglyceride obtained from 400 analyses as references and returned $R$ values in the acceptable range (commercial butter was used as the reference) [34]. Data analysis showed that the absolute values deviated to a minimal extent within the intervals calculated with the official method (AOAC 2005); therefore, this method is applicable for the detection of extraneous fats fraudulently added to butter. In this case, we considered new intervals for the authenticity of butter oils. The official EU method for the detection of animal fats such as lard and tallow is time consuming and tedious, whereas 
the proposed method detects the presence of extraneous vegetable and animal fats in a simple, rapid and precise way, even when the quantity added to butter is minimal (2\% $(\mathrm{w} / \mathrm{w}))$. Adulterations of butter oil of less than $2 \%$ are not monitored [35].

3.6. Summation Method. The summation method consists of setting the area under each triglyceride family equal to unity and adding up all the areas obtained by integration. In this specific case, the butter contained 15 triglyceride families and, consequently, the reference value for the standard butter was precisely 15 (Standard Index). To obtain the index for the butter sample in the analysis, the value of the percentage response for each area of the sample must be divided by the respective percentage in the reference butter for the same family. Compared with the standard, the analysed samples provided an index value between 15.00 and 15.80 , so the proposed index changes with respect to genuine butter types are very small. The addition of small amounts of foreign fat $(<5 \%)$ was not evaluated by this method but used the mathematical method to individually assess each integrated peak. However, adulteration over $10 \%$, producing an index value greater than 15.80 , was easily identified. This method can be used to integrate the graphic method consisting of only overlapping chromatograms and give a qualitative response.

3.7. Comparison of the Area Percentages of Individual Peaks. Another approach to determine the authenticity of butter includes estimation of triglycerides via the integration of individual peaks. One method is based on comparison of the percentages of the individual areas in a butter sample and a standard. This method indicates potential adulteration, detectable as differences in the individual area percentages obtained from integration in the butter sample and standard. For a final assessment of the authenticity of butter with any degree of adulteration, we applied the method of comparison of the individual area percentages to identify the relationships between triglycerides. In vegetable oils, we identified adulteration as low as $1 \%$, as reported using the "graphical method." To detect the presence of added animal fats, specifically lard, the investigation focused on observing differences in the peaks belonging to several families. The graphics overlay of the chromatograms shows the differences between the peaks in an authentic butter sample (a) and a butter sample adulterated with $8 \%$ lard (b), highlighting the individual percentages areas (Figure 6).

3.8. Triglyceride Analysis on a Capillary Column. Separation of the triglycerides in butter on a high-temperature capillary column with a $65 \%$ phenyl methyl silicone stationary phase (RTX 65-TG) allowed application of the Precht method through integration of the triglyceride families, as well as refinement of the GC analysis, because the quantitative determination was performed for individual peaks. This separation was useful for obtaining immediate information from the method comparison chart: butter samples analysed in terms of concentration and GC analysis that

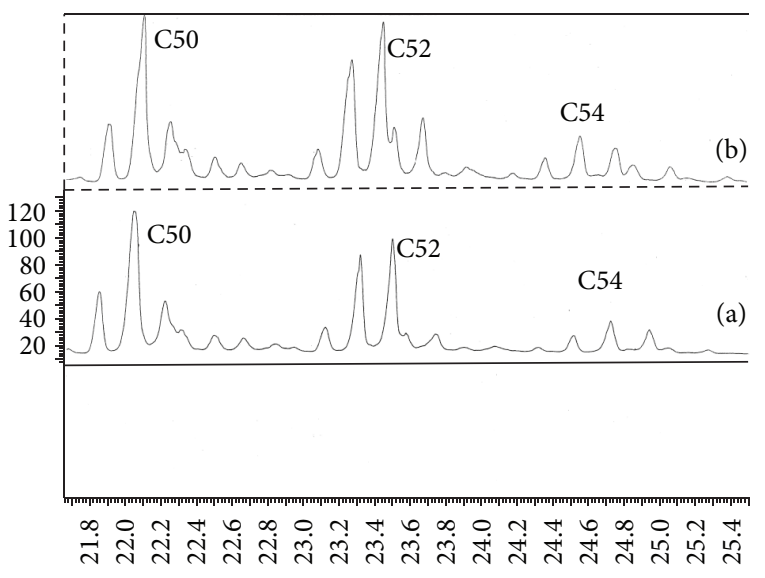

FIgURE 6: Details of triglyceride families with 50, 52, and 54 carbon atoms in authentic butter (a) and butter adulterated with $8 \%$ lard (b).

were identical to a standard were compared to the simple graphical overlay. The graphics overlay of chromatograms provides visual evidence of any peak differences between the reference samples and adulterated samples; specifically, deviation in the GC profile of butter compared to an authentic standard indicates adulteration. The method proposed in this article allows detection of extraneous fats fraudulently added to butter in a simple, rapid, and precise way. Moreover, comparison of literature data on analysed butter samples revealed a narrow variation of characteristic values [34], indicating a close similarity between butter types from various parts of Italy and Europe or between samples taken in different seasons. These conclusions are in contrast to previous data reported in the literature assessing the variability of butter types relative to the provenance or supply of cows.

\subsection{Transesterification of Triglycerides as FA Pentyl Esters.} Figures $7(a)$ and 7 (b) show gas chromatograms of pentyl esters (a) and methyl esters (b) of FAs from the same sample of butter. Numerous studies regarding the appropriate analysis conditions for FAs have revealed the weaknesses of the transesterification of triglycerides as methyl esters due to their high volatility, especially for butyric and caproic acids. For pentyl esters, the volatility of short-chain FAs is negligible, and quantitative determination is consequently more accurate than with methyl esters [36, 37]. For the pentyl esters of FAs, the initial and final column temperatures had to be increased slightly, but the other chromatographic conditions remained unchanged. The transesterification process was very simple and rapid and required the use of sodium metal for the preparation of the catalyst. Sodium pentanoate was prepared in pentanol. When using sodium metal, we took all necessary precautions required for its manipulation, in addition to the normal laboratory safety rules. Sodium metal is widely used in chemical laboratories and can be stored under paraffin indefinitely without causing explosions. The use of metallic sodium requires avoiding contact with water. In our procedure, metallic sodium came into contact with $\mathrm{n}$-hexane and 2-phenyl 


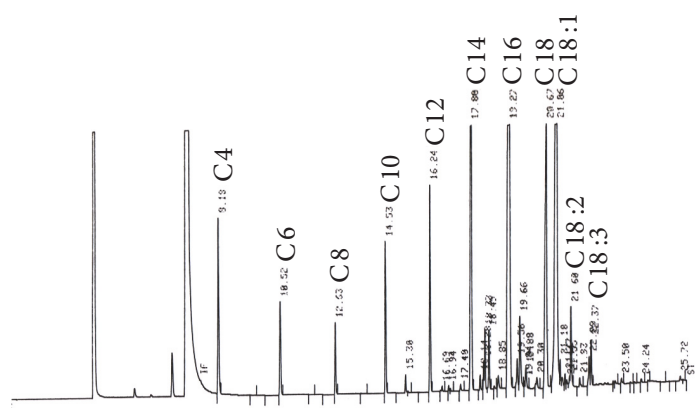

(a)

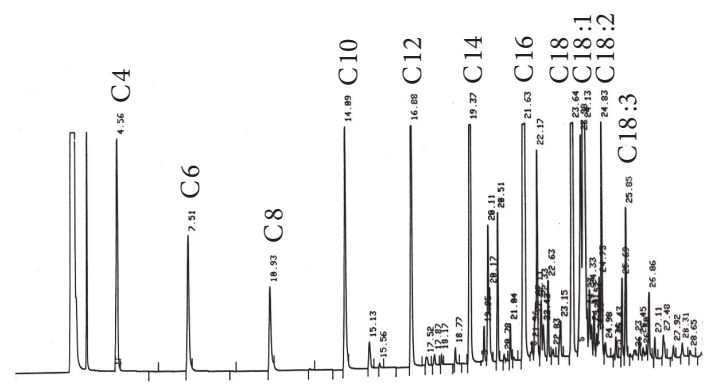

(b)

FIGURE 7: Gas chromatogram of FA pentyl esters (a) and methyl esters (b) in authentic butter.

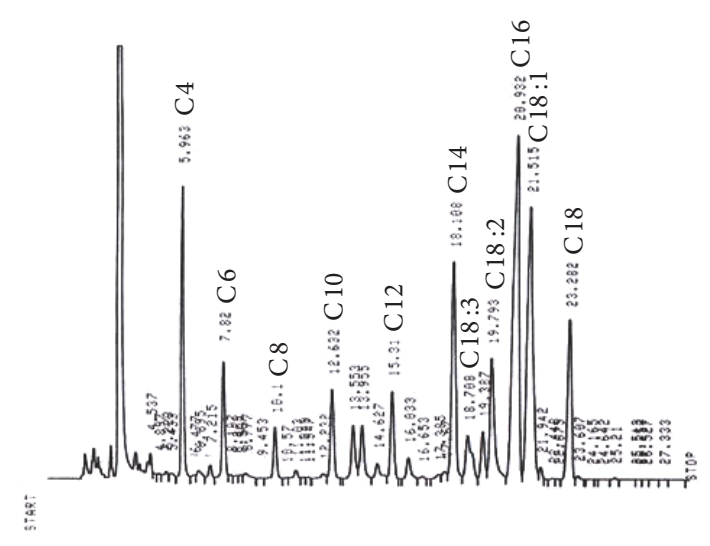

Figure 8: Chromatogram of FA phenethyl esters in butter.

ethanol and was completely consumed by the end of the reaction.

3.10. Transesterification of Triglycerides as Phenethyl Esters of FAs. Figure 8 shows a chromatogram of the phenethyl esters of FAs in butter. All FA phenethyl esters were completely separated from $\mathrm{C} 4$ to $\mathrm{C} 18$. The transesterification reaction occurred as a single stage that did not require saponification. The reaction was quick and occurred at room temperature. Once formed, the phenethyl esters in pentane were added to water and separated. The role of water is to remove all polar compounds that are in the mixture after transesterification reaction, which includes sodium. After the pentane had dried, we added acetonitrile and injected the solution into the
HPLC. This novel transesterification procedure was applied to all of the natural triglyceride mixtures and reduced the degradation of polyunsaturated FAs. The transesterification reaction occurs in a single step, as it does not proceed to saponification reaction, and is fast and usually performed at room temperature, which reduces the degradation of polyunsaturated FAs.

The HPLC applications are the same as those of GC. Both methods have good efficiency, high selectivity, and wide applications, require small amounts of sample, and allow quantitative analyses. However, the HPLC method has the great advantage of being applicable to any liquid mixture, even thermodegradable substances, as vaporization of the sample is not necessary.

Figure 9 presents chromatograms of standard butter and authentic Italian butter, which almost completely overlap, except for the slight differences in the grouping of peaks between $12.5 \mathrm{~min}$ and $17.0 \mathrm{~min}$. The FAs from butyric (C4) to linolenic (C18:3) acids overlapped well. The percentage of each FA in the two butter types was the same (table not shown), and an experimental error of less than $1 \%$ could be calculated for the reproducibility of single FA peaks. Interestingly, the percentages obtained by HPLC were very different from those obtained by GC because the room-temperature conditions of the HPLC analysis lessened the effect of the volatility of short-chain butyric acid and the degradation of unsaturated FAs. Other examples have demonstrated the application of this technique for the analysis of oils.

Figure 10 shows a chromatogram of an olive oil sample and a mixture of $50 \%$ olive oil and $50 \%$ peanut oil as another example of the transesterification of triglycerides as phenethyl esters and the good separation of the FA phenethyl esters obtained after transesterification. The difference in chromatographic separation is due to the different concentrations of FAs between the two matrices. Moreover, the transesterification of triglycerides as phenethyl esters allows application of an alternative method for FA methyl esters, yielding different information about the composition of butter oil.

To summarize, we investigated three methods for analysing fats and oils that predominantly contain triglycerides. The first was based on the Precht method, and triglycerides were analysed using high-resolution GC to obtain compositional information. The practical application of this method is simple and did not require stabilization steps in the GC system, as required by the traditional method. The second method was based on the transesterification of triglycerides as pentyl esters of FAs, in which elongation of the alcohol chain produced heavier esters and increased the length of the ester of butyric acid to nine carbon atoms. Therefore, the difference between butyric acid pentyl ester and butyric acid methyl ester is five carbon atoms. Derivatization as butyl esters still presents minimal volatility and fair solubility in the solution in contact with the underlying transesterified solution, as reported in the literature. In the case of pentyl esters, the recovery of butyric acid was complete, but these data are not reported due to length considerations. The third method was based on the transesterification of fat triglycerides with 2-phenylethanol in a single-stage reaction; 


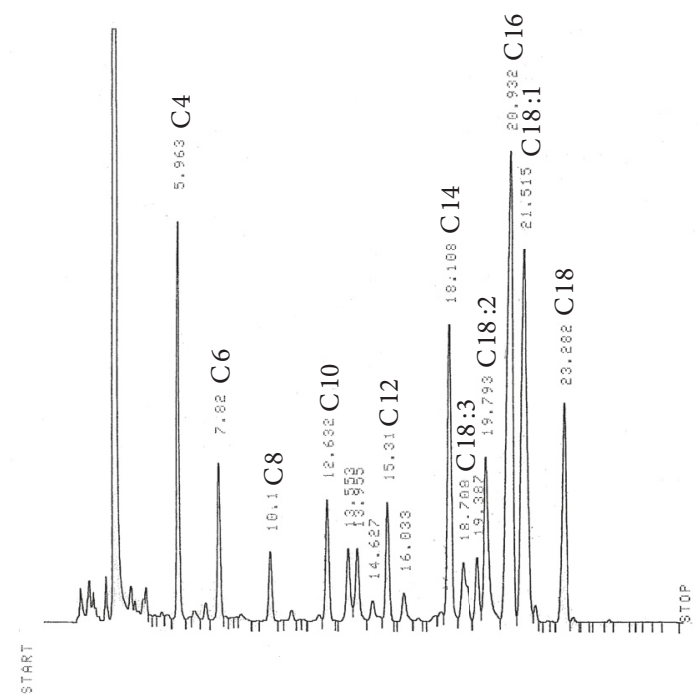

(a)

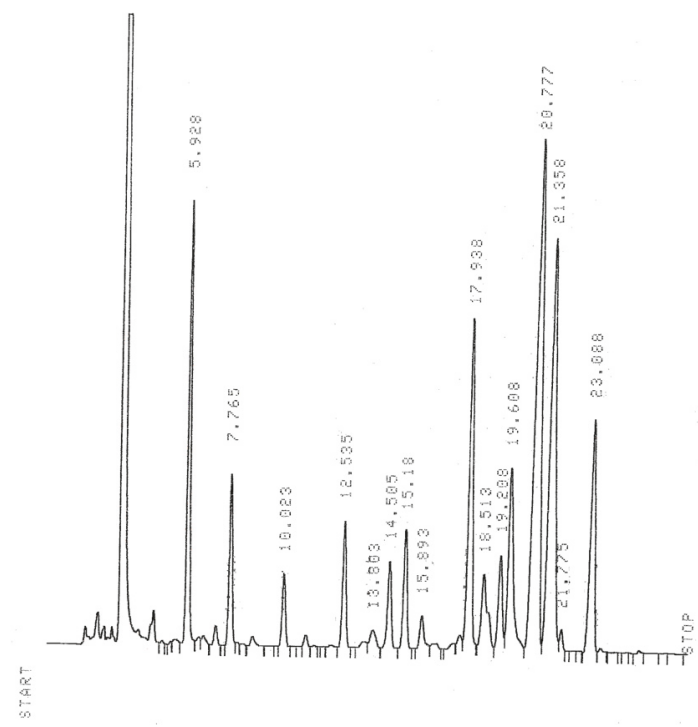

(b)

FIGURE 9: Chromatogram of the FA phenethyl esters in standard butter (a) and authentic Italian butter (b).

2-phenylethanol possesses a chromophore, and the phenethyl esters that were formed were therefore analysed using HPLC with UV detection. The LC analysis was performed at room temperature; as a result, no degradation occurred because of the unsaturated FAs.

\section{Conclusion}

The applicability of three different methodologies for the determination of the authenticity of butter samples has been evaluated in this manuscript. These approaches are based on chromatographic methods (GC and LC) for the determination of triglycerides families (direct analysis of the samples after dilution) and individual FAs as pentyl and phenethyl esters. The first method enables the analysis of triglycerides by

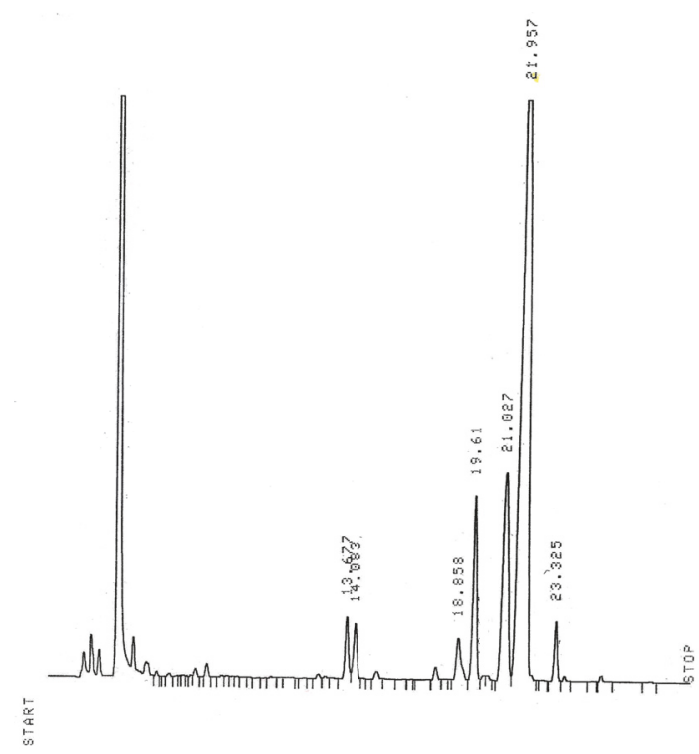

(a)

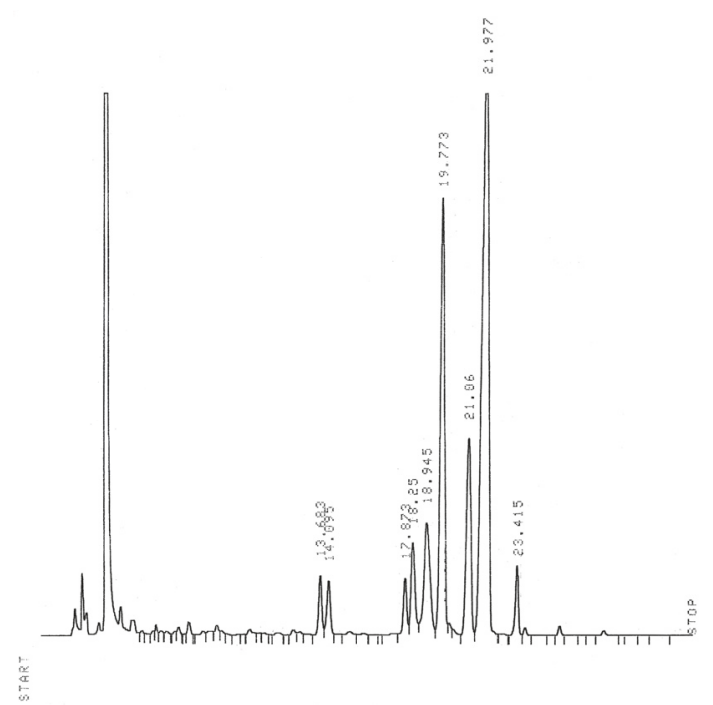

(b)

FIGURE 10: Chromatogram of FA phenethyl esters in olive oil (a) and a mixture of olive and peanut oil $(50: 50)$ (b).

techniques such as high-resolution GC and the application of the official method of Precht to gain additional information about the triglyceride composition because it is based on the best obtainable separation, which can currently be obtained using a high-temperature capillary column (RTX 65-TG). This method is simple in its practical application and does not require the stabilization phase of the GC system that is required in the official method; moreover, a simple method based on chromatogram overlap can be used to qualitatively evaluate the authenticity of butter oil. The second method is based on the transesterification of triglycerides as FA pentyl esters; the elongation of the alcohol chain produced heavier esters and increased the length of the ester of butyric acid to nine carbon atoms. Derivatization as butyl esters 
still presents minimal volatility and fair solubility in the solution in contact with the underlying transesterification solution, as reported in the literature. In the case of pentyl esters, the recovery of butyric acid was complete. The third method involves the transesterification of the triglycerides in fat with 2-phenylethanol (catalysed by 2-phenylethanoate) in a single stage; 2 -phenylethanol possesses a chromophore, and the phenethyl esters that are formed are analysed by HPLC with UV detection. The analysis by LC, carried out at room temperature, is not affected by any degradation of unsaturated FAs that may occur. Finally, the proposed methods can pave the way for new investigations of the triglyceride and FA composition of fats and oils because they use high-resolution chromatographic techniques and minimize the errors associated with the analysis method.

\section{Additional Points}

Practical Applications. The determination of triglycerides and/or FAs in food is an interesting topic in food chemistry. Most of the results included in this manuscript focus on the direct analysis of triglycerides for the detection of butter adulteration, but the proposed methods could also be used with other fat matrices. Furthermore, the analysis methods described in this work, in addition to being rapid and inexpensive compared to the more sophisticated techniques existing today, such as mass spectrometry, can improve and/or integrate the analytical results obtained with traditional chromatographic methods in both the liquid (HPLC) and gas (GC) phase.

\section{Conflicts of Interest}

The authors declare no conflicts of interest regarding the publication of this paper.

\section{References}

[1] E. Tvrzicka, L.-S. Kremmyda, B. Stankova, and A. Zak, "Fatty acids as biocompounds: their role in human metabolism, health and disease-a review. Part 1: classification, dietary sources and biological functions," Biomedical Papers, vol. 155, no. 2, pp. 117130, 2011.

[2] D. Precht, "Detection of adulterated milk fat by fatty acid an triglyceride analysis," Fat Science Technology, vol. 93, no. S4, pp. 538-544, 1991.

[3] J. Molkentin, "Detection of foreign fat in milk fat from different continents by triacylglycerol analysis," European Journal of Lipid Science and Technology, vol. 109, no. 5, pp. 505-510, 2007.

[4] W. W. Christie, Lipid Analysis: Isolation, Separation, Identification and Structural Analysis of Lipids, Pergamon Press, Oxford, UK, 1982.

[5] AOCS, "Sampling and analysis of commercial fats and oils," in Official Methods and Recommended Practices of the AOCS, V. C. Mehlenbacher and E. M. Sallee, Eds., pp. Ce2-Ce66, Amer Oil Chemists Society, Champaign, Ill, USA, 2009.

[6] B. W. Kail, D. D. Link, and B. D. Morreale, "Determination of free fatty acids and triglycerides by gas chromatography using selective esterification reactions," Journal of Chromatographic Science, vol. 50, no. 10, pp. 934-939, 2012.
[7] C. Ruiz-Samblás, A. González-Casado, and L. CuadrosRodríguez, "Triacylglycerols determination by high-temperature gas chromatography in the analysis of vegetable oils and foods: a review of the past 10 years," Critical Reviews in Food Science and Nutrition, vol. 55, no. 11, pp. 1618-1631, 2015.

[8] R. Rombaut, N. De Clercq, I. Foubert, and K. Dewettinck, "Triacylglycerol analysis of fats and oils by evaporative light scattering detection," JAOCS, Journal of the American Oil Chemists' Society, vol. 86, no. 1, pp. 19-25, 2009.

[9] M. Buchgraber, F. Ulberth, and E. Anklam, "Method validation for detection and quantification of cocoa butter equivalents in cocoa butter and plain chocolate," Journal of AOAC International, vol. 87, no. 5, pp. 1164-1172, 2004.

[10] P. A. Guy and F. Fenaille, "Contribution of mass spectrometry to assess quality of milk-based products," Mass Spectrometry Reviews, vol. 25, no. 2, pp. 290-326, 2006.

[11] N. Ogrinc, I. J. Košir, J. E. Spangenberg, and J. Kidrič, “The application of NMR and MS methods for detection of adulteration of wine, fruit juices, and olive oil. A review," Analytical and Bioanalytical Chemistry, vol. 376, no. 4, pp. 424-430, 2003.

[12] F. Aranda, S. Gómez-Alonso, R. M. Rivera Del Álamo, M. D. Salvador, and G. Fregapane, "Triglyceride, total and 2-position fatty acid composition of Cornicabra virgin olive oil: comparison with other Spanish cultivars," Food Chemistry, vol. 86, no. 4, pp. 485-492, 2004.

[13] L. M. Reid, C. P. O’Donnell, and G. Downey, "Recent technological advances for the determination of food authenticity," Trends in Food Science and Technology, vol. 17, no. 7, pp. 344-353, 2006.

[14] A. I. Carrapiso and C. Garcia, "Development in lipid analysis: Some new extraction techniques and in situ transesterification," Lipids, vol. 35, no. 11, pp. 1167-1177, 2000.

[15] J. Eras, F. Montañes, J. Ferran, and R. Canela, "Chlorotrimethylsilane as a reagent for gas chromatographic analysis of fats and oils," Journal of Chromatography A, vol. 918, no. 1, pp. 227-232, 2001.

[16] K.-S. Liu, "Preparation of fatty acid methyl esters for gaschromatographic analysis of lipids in biological materials," Journal of the American Oil Chemists' Society, vol. 71, no. 11, pp. 1179-1187, 1994.

[17] G. Nota, D. Naviglio, R. Romano, V. Sabia, and S. Spagna Musso, "Evaluation and improvement of transesterification methods of triglycerides," Analytical Letters, vol. 31, no. 14, pp. 2499-2512, 1998.

[18] N. Dubois, G. Barnathan, J.-P. Gouygou, and J.-P. Bergé, "Gas chromatographic behavior of fatty acid derivatives for mass spectrometry on low-polarity capillary columns," European Journal of Lipid Science and Technology, vol. 111, no. 7, pp. 688697, 2009.

[19] G. Dobson and W. W. Christie, "Spectroscopy and spectrometry of lipids-part 2. Mass spectrometry of fatty acid derivatives," European Journal of Lipid Science and Technology, vol. 104, pp. 36-43, 2002.

[20] W. W. Christie, "Some recent advances in the chromatographic analysis of lipids," Analusis, vol. 26, no. 3, pp. M34-M40, 1998.

[21] W. W. Christie, "Gas chromatography-mass spectrometry methods for structural analysis of fatty acids," Lipids, vol. 33, no. 4, pp. 343-353, 1998.

[22] V. Spitzer, "Structure analysis of fatty acids by gas chromatography-low resolution electron impact mass spectrometry of their 4,4-dimethyloxazoline derivatives-a review," Progress in Lipid Research, vol. 35, no. 4, pp. 387-408, 1996. 
[23] W. W. Christie, E. Y. Brechany, S. B. Johnson, and R. T. Holman, "A comparison of pyrrolidide and picolinyl ester derivatives for the identification of fatty acids in natural samples by gas chromatography-mass spectrometry," Lipids, vol. 21, no. 10, pp. 657-661, 1986.

[24] G. Dobson and W. W. Christie, "Structural analysis of fatty acids by mass spectrometry of picolinyl esters and dimethyloxazoline derivatives," TrAC - Trends in Analytical Chemistry, vol. 15, no. 3, 1996.

[25] F. Destaillats and P. Angers, "One-step methodology for the synthesis of FA picolinyl esters from intact lipids," Journal of the American Oil Chemists' Society, vol. 79, no. 3, pp. 253-256, 2002.

[26] J. T. G. Hamilton and W. W. Christie, "Mechanisms for ion formation during the electron impact-mass spectrometry of picolinyl ester and 4,4-dimethyloxazoline derivatives of fatty acids," Chemistry and Physics of Lipids, vol. 105, no. 1, pp. 93$104,2000$.

[27] G. Dobson and J. L. Sebedio, "Monocyclic dienoic fatty acids formed from $\gamma$-linolenic acid in heated evening primrose oil," Chemistry and Physics of Lipids, vol. 97, no. 2, pp. 105-118, 1999.

[28] N. Dubois, C. Barthomeuf, and J.-P. Bergé, "Convenient preparation of picolinyl derivatives from fatty acid esters," European Journal of Lipid Science and Technology, vol. 108, no. 1, pp. 28-32, 2006.

[29] J. Y. Zhang, Q. T. Yu, B. N. Liu, and Z. H. Huang, “Chemical modification in mass spectrometry IV-2-alkenyl-4,4-dimethyloxazolines as derivatives for the double bond location of long-chain olefinic acids," Biomedical \& Environmental Mass Spectrometry, vol. 15, no. 1, pp. 33-44, 1988.

[30] I. Molnar, "Computerized design of separation strategies by reversed-phase liquid chromatography: development of DryLab software," Journal of Chromatography A, vol. 965, no. 1-2, pp. 175-194, 2002.

[31] N. L. Lévêque, S. Héron, and A. Tchapla, "Regioisomer characterization of triacylglycerols by non-aqueous reversed-phase liquid chromatography/electrospray ionization mass spectrometry using silver nitrate as a post column reagent," Journal of Mass Spectrometry, vol. 45, no. 3, pp. 284-296, 2010.

[32] H.-G. Janssen, W. Boers, H. Steenbergen, R. Horsten, and E. Flöter, "Comprehensive two-dimensional liquid chromatography X gas chromatography: evaluation of the applicability for the analysis of edible oils and fats," Journal of Chromatography A, vol. 1000, no. 1-2, pp. 385-400, 2003.

[33] P. Dugo, O. Favoino, P. Q. Tranchida, G. Dugo, and L. Mondello, "Off-line coupling of non-aqueous reversed-phase and silver ion high-performance liquid chromatography-mass spectrometry for the characterization of rice oil triacylglycerol positional isomers," Journal of Chromatography A, vol. 1041, no. 1-2, pp. 135-142, 2004.

[34] D. Naviglio and C. Raia, "Application of a hrgc method on capillary column RTX ${ }^{\circledR}$ 65-TG for triglyceride analysis to monitor butter purity," Analytical Letters, vol. 36, no. 14, pp. 3063-3094, 2003.

[35] M. A. De La Fuente and M. Juárez, "Authenticity assessment of dairy products," Critical Reviews in Food Science and Nutrition, vol. 45, no. 7-8, pp. 563-585, 2005.

[36] W. W. Christie, Gas Chromatography and Lipids, Oily Press, Ayr, UK, 1989.

[37] W. W. Christie and X. Han, Lipid Analysis-Isolation, Separation, Identification and Lipidomic Analysis, Oily Press, Bridgwater, UK, 2010. 

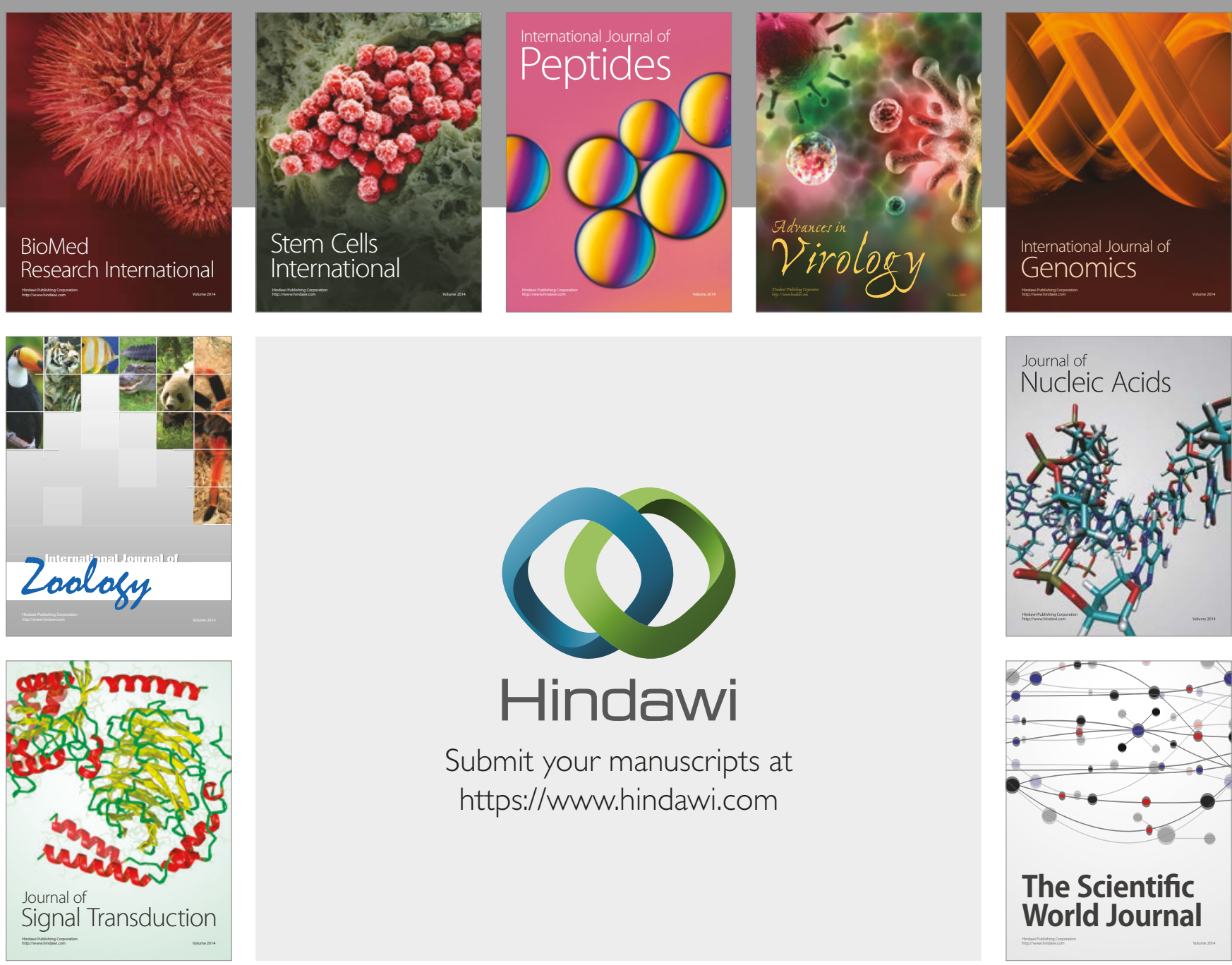

Submit your manuscripts at

https://www.hindawi.com
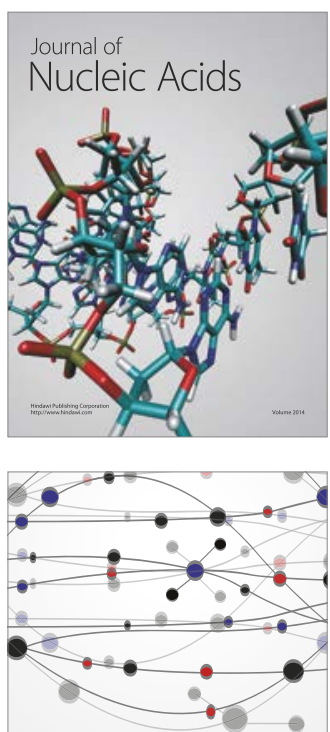

The Scientific World Journal

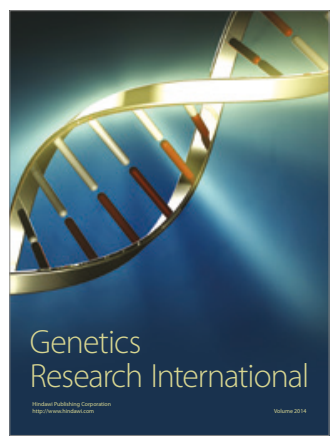

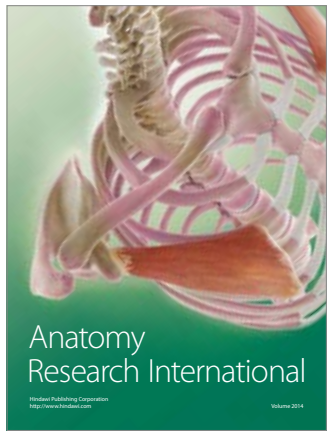

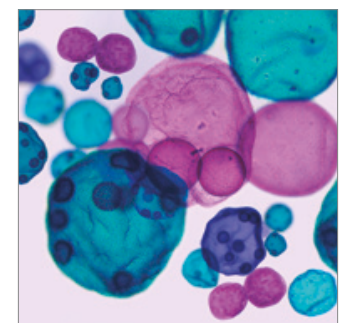

International Journal of Microbiology
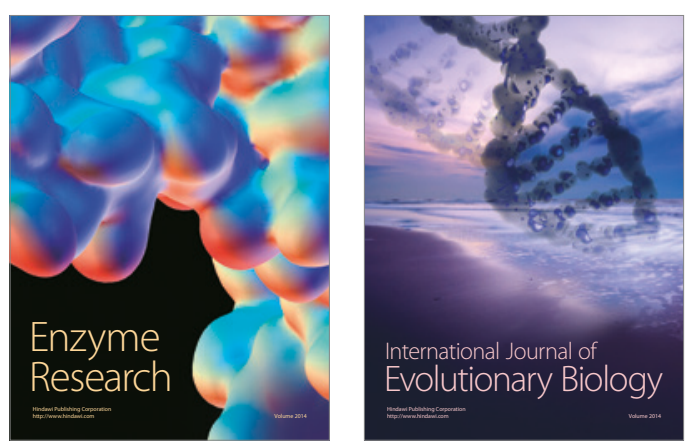
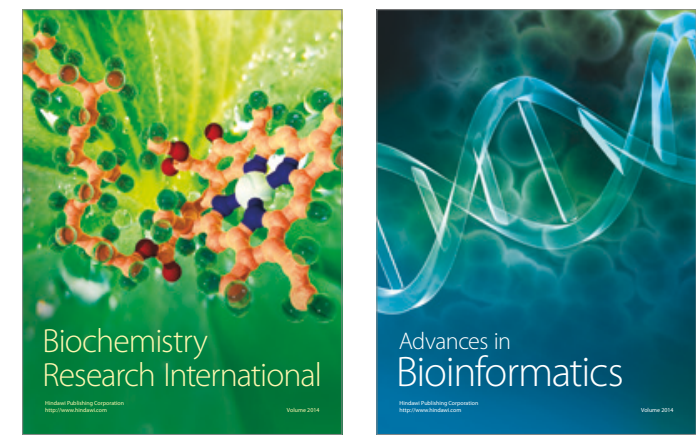

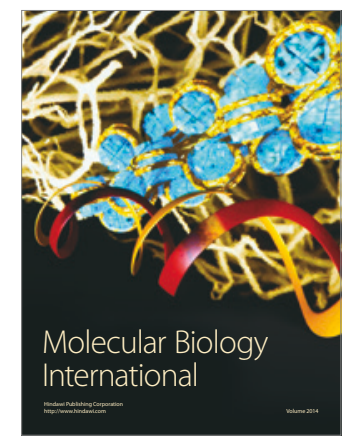

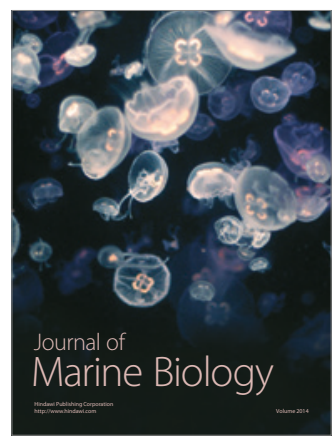

